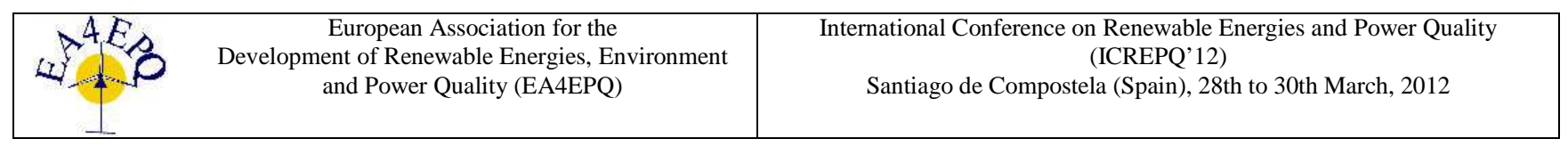

\title{
Evolutionary algorithm application to medium voltage power system restoration
}

\author{
J. Csatár ${ }^{1}$, A. Dán SM IEEE ${ }^{2}$, I. Vokony ${ }^{3}$ \\ ${ }^{1}$ Student at the Department of Electric Power Engineering \\ Budapest University of Technology and Economics \\ Egry József u. 18, H-1111 Budapest (Hungary) \\ e-mail: csatar.janos@gmail.com \\ ${ }^{2}$ Professor at the Department of Electric Power Engineering \\ Budapest University of Technology and Economics \\ Egry József u. 18, H-1111 Budapest (Hungary) \\ Phone: +36 1463 3019, e-mail: dan.andras@ vet.bme.hu \\ ${ }^{3} \mathrm{PhD}$ Student at the Department of Electric Power Engineering \\ Budapest University of Technology and Economics \\ Egry József u. 18, H-1111 Budapest (Hungary) \\ Phone: +36 1463 3030, e-mail: vokony.istvan@vet.bme.hu
}

\begin{abstract}
.
The voltage quality is one of the most important issue in power system. The distribution network has to keep up with the growing consumption and increasing reliability requirements. In many cases supply disturbance is caused by faults on medium voltage distribution networks. Throughout system restoration many switching operation are needed, figuring out the optimal ones is a demanding task. In this paper a genetic algorithm based method is proposed, which can reduce the outage time in those areas where supply can be restored while the repairing is carried out on the failed section.
\end{abstract}

\section{Key words}

Genetic algorithm, restoration, medium voltage distribution system.

\section{Introduction}

Continuous supply is one of the most important factor in voltage quality. In many cases supply disturbances are caused by faults on medium voltage systems. Throughout the restoration process many switching operation are needed (eg. To isolate the faulted section). Often it is not trivial how to provide electricity to those customers that are not affected directly by the isolated section. The proposed method helps overcoming this problem by suggesting an optimal switching procedure.

Previous works in this field includes expert systems[1], heuristic methods[2], neural networks[3] and evolutionary algorithms[4]. They all have their advantages and disadvantages. Most of them focuses on one subject and not consider the others, the referred articles gives a more comprehensive solution.

Designing the rules for expert systems are tedious, have to be customized for every network and needs great proficiency, expert knowledge, but the decision making is closer to human thinking and thus easier to see the reason behind the result. Neural networks too have to be taught for every network and it doesn't indicate if the result is valid or not. Heuristic methods don't offer optimal solutions, although they can be close to it. Evolutionary algorithm application to this problem is relatively new, promises the chance to find optimal solution and can be more flexible than the others. Instead of focusing on one sub problem, the proposed method tries to offer a complete solution.

\section{Genetic algorithm}

Genetic algorithms[5] are nature inspired computing method in which the optimal solution is searched for through selection and reproduction (just like how the animals adapt to their environment in evolution). Populations (a group of individuals) follow each other, the best individuals are selected to leave offspring (with crossover and mutation). The next population will consist of these offspring (if elitism is present than some of the best parents will also be included). To determine which individual is better than the other fitness value is used. An individual consists of a chromosome (a set of genes, which stores the solution) and the fitness value. 
If constraints have to be handled than other techniques are needed such as ranking. This way the fitness values and constraint violations can be "merged" into one indicator.

\section{The solving process}

The proposed method separates the problem into two parts, both handled by ranked genetic algorithms. Ranking helps to handle constraints in search space, thereby only local knowledge of the problem is needed.

\section{A. Objectives and constraints}

At system restoration the followings are desired:

- Shortest possible interruption,

- Minimum energy not supplied,

- And minimum number of consumers affected.

And also have some condition:

- Radial topology should be maintained,

- Voltage level should be in the defined threshold,

- Over-current should be avoided.

\section{B. Simplification}

The solving process has been separated into two parts, thus the complexity is reduced to a more manageable level. Firstly the desirable network topology is determined, secondly the optimal switching order is decided.

Moreover the initial network is reduced: usually the area where switching is needed can be predicted, the rest of the network can be replaced along the tie lines by generation units which has corresponding capacity.

If only remote controllable switching operations are desired, the manual ones are removed, also switches can be locked in opened or closed position and are removed from the genes.

\section{Optimal network topology}

The first part is a multi-objective, multi-constrained, non continuous problem for which an algorithm based on NSGA-2[6] is developed and implemented in MATLAB. With NSGA-2 true multi-objective optimization can be performed with the help of distance value and ranking which rests on non dominated sorting. Distance helps to promote diversity in the population, it measures how "close" the individuals in a population are.

Constraints handling is included through domination. One individual dominates the other if it is better in every fitness value, or - in case of constraint violation - it violates the constraints to a lesser extent.

Crowded tournament selection is used: two individual are picked, the one with the better rank or - in case of both are in the same rank - the one with the greater distance is the winner.

The minimization objectives used:

- Number of switching,

- Lost supplied power.
The constraints used:

- Radial topology (included in gene coding - hard constraint)

- Voltage threshold

- Current threshold

The constraints are calculated with load flow[7]: where the voltage drop was too high or overload occurred, its violation percentage values are summed.

String representation is not the usual binary where every switch has its corresponding gene (which value indicates whether it is in opened or closed state), but permutation based. Every switch has its number and the chromosome is a permutation of them. A marker gene is also included to handle those situations, where some consumers can't be supplied.

The statuses of the switches are created during decoding:

- At the starting every switch is opened.

- Then from the first gene on, every one is inspected: it is closed if it doesn't violate some rules (ie. Loop in network, or parallel supply), otherwise it remains opened.

- If the marker gene is reached the rest of the switches stay in opened position.

This way every possible permutation leads to a valid configuration, a repairing operator is not needed opposed to binary representation. The search space is increased, but is also more homogenous.

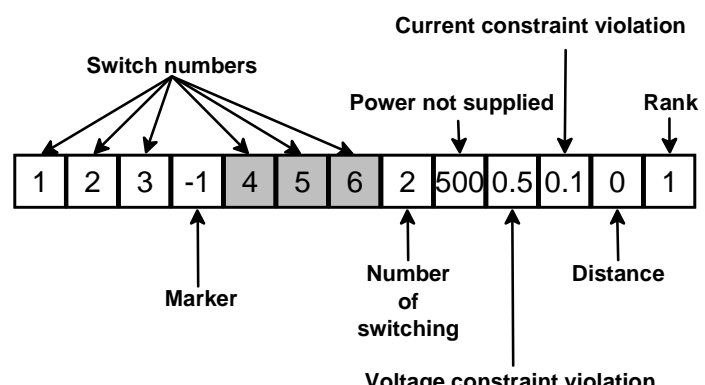

Fig. 1. How an individual is stored, the switches marked by gray background stay opened at every evaluation.

For this representation the following mutation and crossover proved to be efficient:

\section{Mutation}

Every individual undergoes mutation (elitism helps to preserve good solutions). Has one parameter that from the initial 0.5 linearly decrease to 0 with generations passing. This describes the chance that one gene mutates. For example if the value is 0.5 than on average half of the chromosome will change, if it is $1 / n$ (where $n$ is the number of the genes) than on average one gene will change in a chromosome.

If a gene is picked for mutation it is swapped with a random other gene in the chromosome. 


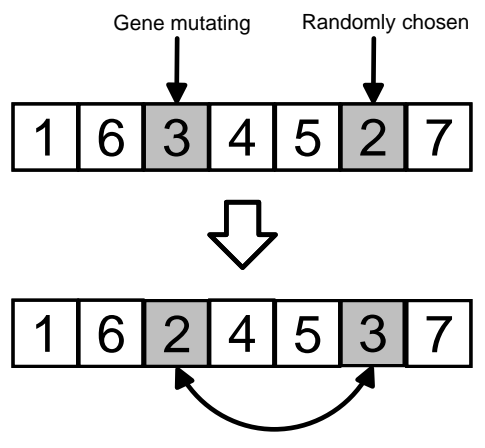

Fig. 2. Mutation

\section{Crossover}

Also has one parameter: the chance of a pair undergoes crossover (if not, they are simply copied to their offspring). A random section of the chromosome is picked in the same place of both parents. Their contents are marked in the other parent. The parent's section goes to their offspring, the rest of the spaces are filled by the other parent's non-marked genes in order.

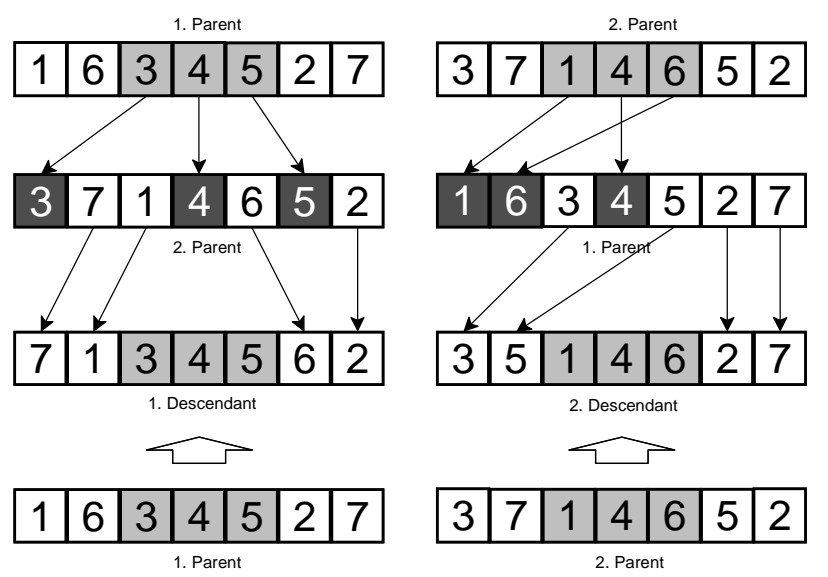

Fig. 3. Crossover

The final solution can be chosen by either objective a combination of both, or other measurement.

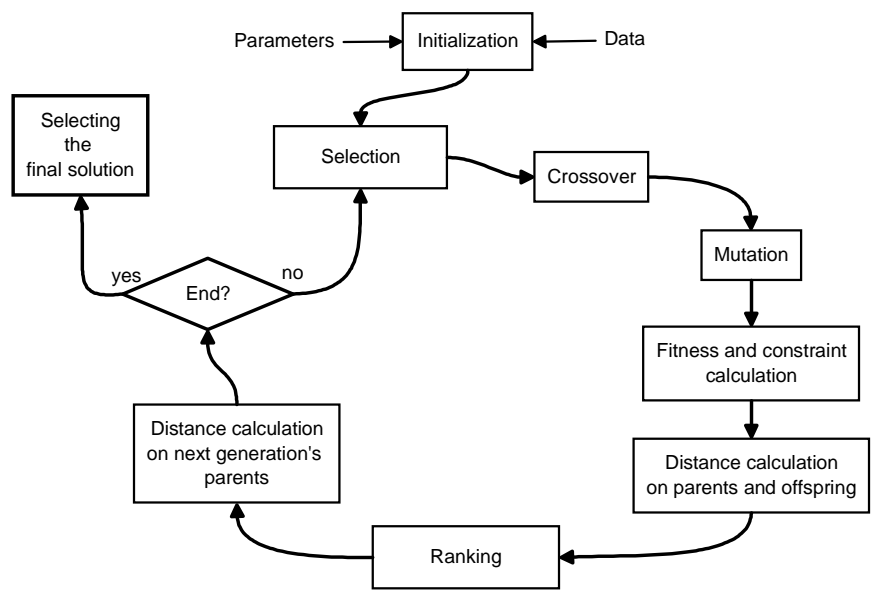

Fig. 4. Overview of the first part's working mechanism.

\section{Optimal switching sequence}

The second part is used for optimizing the switching task received from the first part. Multiple teams can be dispatched, thus parallel switching is possible. The objective here is to minimize the required time. Only one constraint is present: switching precedence in some cases (for example to avoid overloading during switching).

A ranked genetic algorithm is developed for this purpose. String representation is also permutation based: every switch has a number (like in the first part) and there are some marker genes to assign tasks to teams.

The ranking procedure is called Dynamic Stochastic Ranking [8], and it is based on bubble sorting. If none of the individuals violate the constraint than it is ordinary bubble sorting by fitness value. If constraint violation occurs than there is a chance that the comparison of two individual is only based on fitness value, otherwise the one with the lesser constraint violation will get better rank. This parameter is decreased linearly with generations passing from an initial 0.45 to 0 following the suggestion from the original article.

The mutation and crossover operator from the first part proved to be efficient here as well.

\section{Results}

The results on a small test system are introduced here. Some test cases:

- Everything is in normal condition except consumer number 7: its load has been increased, therefore the voltage drop is too high. The suggested solution was to open switch 9 and close switch 14 (in this order), with this supply point 9 can serve the load.

- Everything is in normal condition except supply point 1: its capacity has been decreased, therefore it is overloaded. The suggested solution was to supply consumer 7 and 6 from the supply point 9 . Switching task: open switch 6 and close switch 14 in this order.

- A fault before switch 1, therefore consumers 28 are without supply. The suggested solution (preferred the least power not supplied): consumer 4-5 was supplied from point 29; consumer 6, 7 and 8 was supplied from point 9; consumer 2 and 3 remains without power. Switching task: close switches 13, 12 and 20 (number 1 was automatically opened by protection device), open: switches 2, 3, 6 and 8 . If one team is available than the order is 8,6 , (remotely close 12, 13) 3, 2, (remotely close $20)$. If two teams can be dispatched than team one opens 6,8 , (remotely close 12,13 ) team two opens 3, 2, (remotely close 20). Thus the time needed to supply the consumers 2 and 3 almost halves.

- Switch 22 is broken and locked in opened position. The suggested solution was: consumer 23 can be supplied with the closing of switch 21 (supply point 1 is near its maximum load 
otherwise switch 20 and 18 would be equally good solution).

As it can be seen from the figures bellow, good converge was obtained. The smaller the problem the fewer the required generations are. For another test system - that has 138 switches on the network - the required generations to obtain good solutions increased linearly, and the time that one generation takes also increased around linearly. Of course the performance relies on the properties of the network examined.

The number of switching is following the trends of the power not supplied.

For the first part, out of 30 trials, the optimal solution was achieved 28 times for the root fault. The remaining two were 8 switches with $500 \mathrm{KW}$ power not supplied, and 7 switches with $550 \mathrm{KW}$ power not supplied - not too bad compared to the best. And all of the 30 runs reached optimal solution for the broken switch problem and for the overstressed system relief problems.

The switching task optimization part also shows good convergence and found 28-29 optimal solution out of 30 trials.

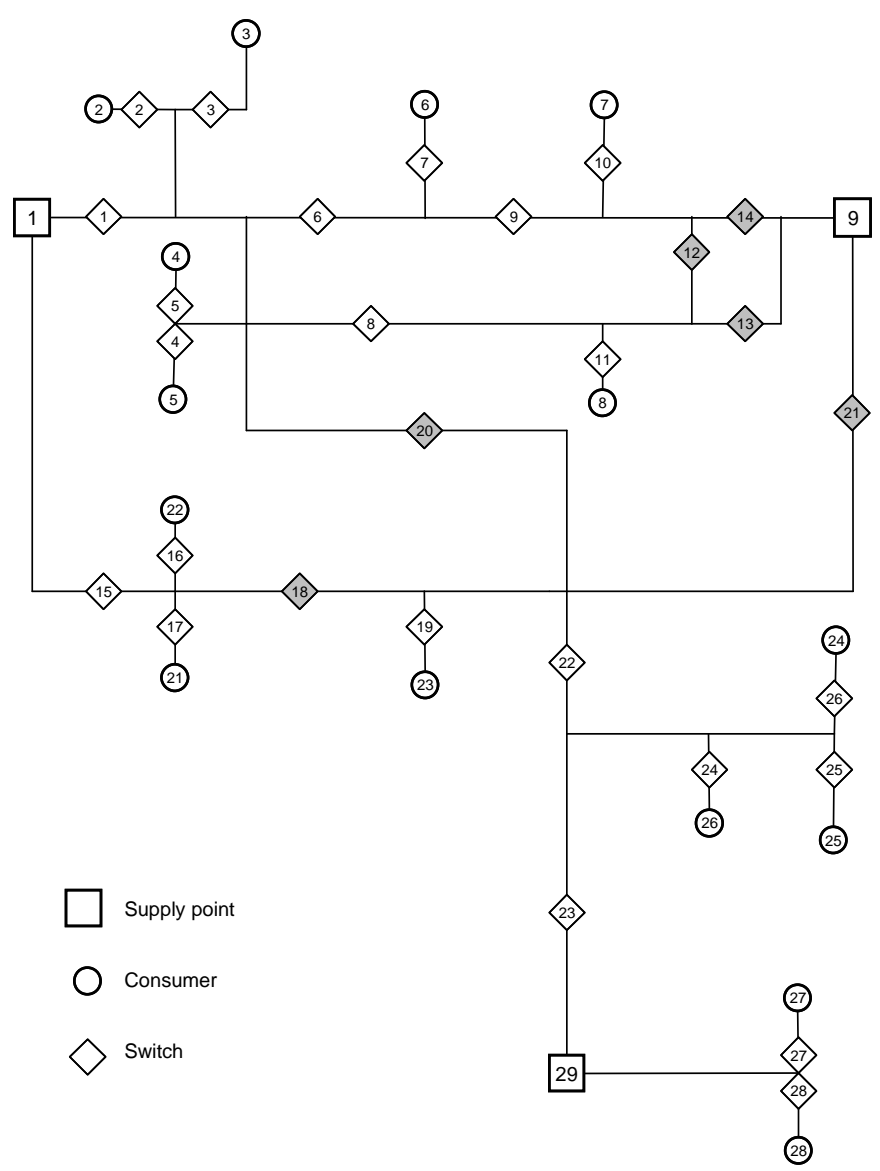

Fig. 5. The test system, normally open tie switches (remote controllable) are marked by gray background

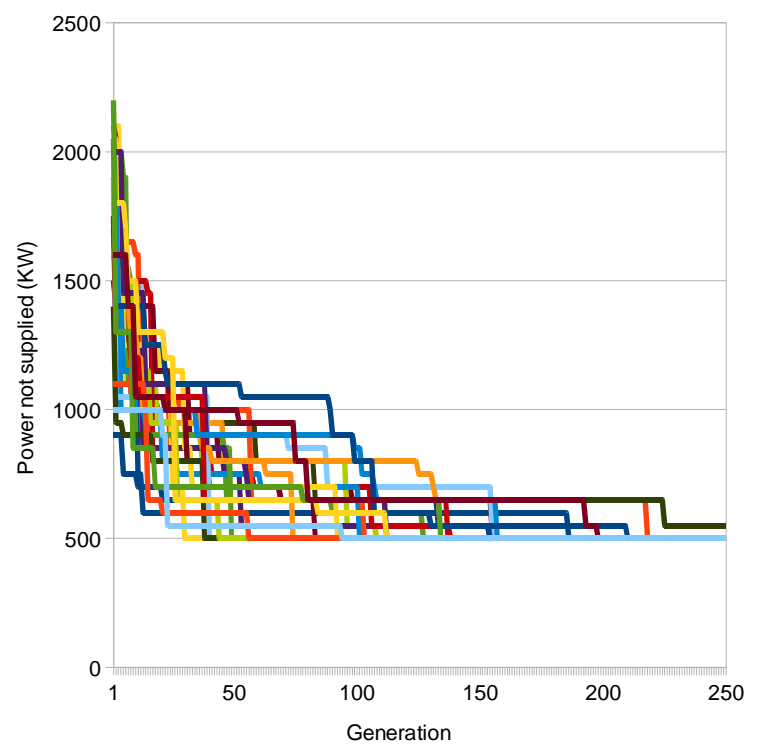

Fig. 6. Best individual in population - power not supplied, first part, 30 trial, case: fault before switch 1

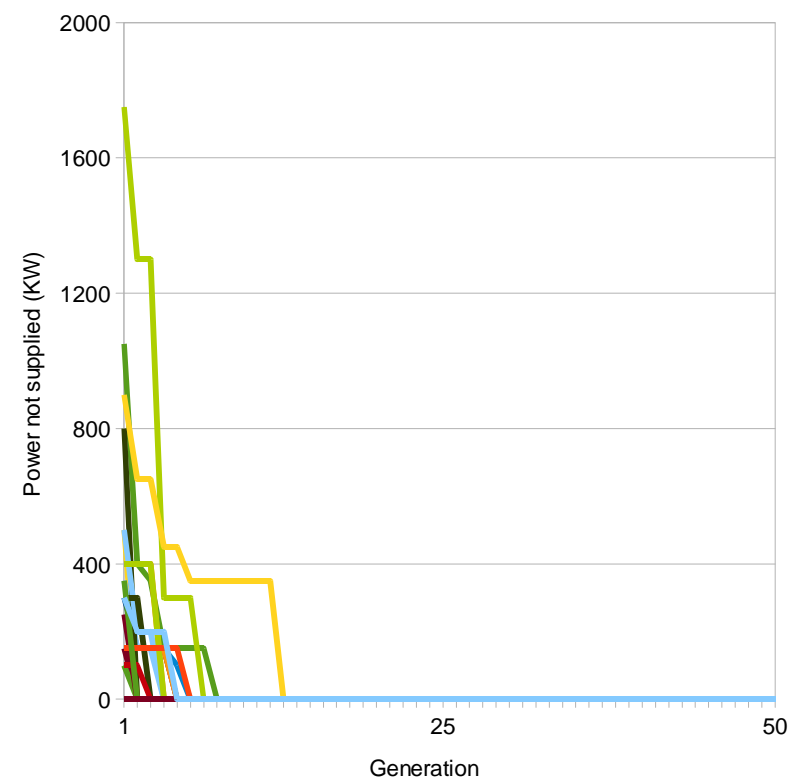

Fig. 7. Best individual in population - power not supplied, first part, 30 trial, case: switch 22 broken

Within a minute multiple evaluations can be executed, therefore the chance to obtain optimal solution greatly improve.

Great speedup might be achieved by parallel execution at larger systems and/or by implementing the method in a lower level programming language.

\section{Conclusion}

The proposed method is fast, flexible and doesn't need tedious parameterization for every network. Combined with other methods for locating faults, the majority of the affected customers can be resupplied within minutes using remote controllable switches.

More tests are planned to discover the limits of usability. 


\section{Acknowledgement}

This work is connected to the scientific program of the "Development of quality-oriented and harmonized R+D+I strategy and functional model at BME" project, supported by the New Hungary Development Plan (Project ID: TÁMOP 4.2.1/B-09/1/KMR-2010-0002).

\section{References}

[1] H.-T. Yang and C.-M. Huang. Distribution system service restoration using fuzzy Petri-net models. International Journal of Electrical Power and Energy Systems, 24(5):395-403, June 2002.

[2] S. Dimitrijevic and N. Rajakovic. An innovative approach for solving the restoration problem in distribution networks. Electric Power Systems Research, 81(10):1961-1972, October 2011.

[3] C.-M. Huang, C.-T. Hsieh, and Y.S. Wang. Evolution of radial basic function neural network for fast restoration of distribution systems with load variations. International Journal of Electrical Power and Energy Systems, 33(4):961-968, May 2011.

[4] S. Siqing, M. Zhigang, W. Jing, and G. Nan. Distribution network fault restoration based on improved adaptive genetic algorithm. In 2009 Second International Conference on Intelligent Computation Technology and Automation, pages 318-321, October 2009.

[5] D.E. Goldberg. Genetic Algorithms in Search, Optimization and Machine Learning. Kluwer Academic Publishers, 1989.

[6] K. Deb, A. Pratap, S. Agarwal, and T. Meyarivan. A fast and elitist multiobjective genetic algorithm: NSGA-II. IEEE Trans. Evol. Comput., 6(2):182-197, April 2002.

[7] D. Shirmoharmnadi, H. W. Hong, A. Semlyen, and G. X. Luo. A compensation-based power flow method for weakly meshed distribution and transmission networks. IEEE Transactions on Power Systems, 3(2):753-762, May 1988.

[8] M. Zhang, W. Luo, and X. Wang. Differential evolution with dynamic stochastic selection for constrained optimization. Information Sciences, 178(15):3043-3074, January 2008. 